\title{
Primeros 230 casos en cirugía general asistida por robot por un solo grupo quirúrgico en México
}

\author{
First 230 cases in robotic assisted general surgery by a single surgical group in Mexico \\ Javier Alberto Kuri Osorio, ${ }^{*}$ Juan José Solórzano Adame, ${ }^{*}$ Manuel Preben Aguirre García, ${ }^{*}$ \\ Francisco Iván Galeana Nogueda, ${ }^{*}$ Karla Ivonne Luján Mendoza* \\ * Centro de Cirugía Robótica. Hospital Ángeles Pedregal. Ciudad de México.
}

\section{RESUMEN}

Objetivos: La cirugía robótica cada día se encuentra más disponible y en algunos ejemplos supera a los otros abordajes quirúrgicos, brindando mejores resultados para los pacientes. El presente estudio describe la experiencia de un solo grupo quirúrgico en cirugía general, en dos centros hospitalarios privados de tercer nivel en la Ciudad de México. Material y métodos: De enero de 2016 a agosto de 2020, 230 pacientes con diferentes enfermedades se sometieron a intervención robótica dentro de la especialidad de cirugía general, las operaciones se realizaron por un solo cirujano calificado. Los resultados quirúrgicos se registraron en una base de datos. Resultados: Se sometieron a cirugía robótica 230 pacientes, $44 \%$ (102) fueron mujeres y $56 \%$ (128) fueron hombres, cuya edad promedio era de 56.5 años, con un rango de 19-88 años. La cirugía de hiato fue la más realizada en este periodo, acumulando 100 intervenciones ( $43.5 \%$ del total), seguida por las plastias inguinales, que sumaron $98(42.6 \%)$, plastias ventrales $13(5.7 \%)$, colectomías $10(4.3 \%)$, colecistectomías $4(1.7 \%)$, gastrectomías $3(1.4 \%)$, resección de lesión duodenal $1(0.4 \%)$ y restitución del tránsito intestinal con resección de fístula colovaginal 1 . El tiempo quirúrgico promedio fue de 178.4 min. Sólo existió una conversión a cirugía abierta y no se reportó ninguna complicación mayor con una morbilidad de $4.7 \%$. Conclusiones: La cirugía robótica se está realizando con éxito y ofrece resultados comparables a los centros de excelencia internacional y existe una línea del tiempo en la experiencia del cirujano robótico que permite, conforme acumula un número de eventos y experiencia en el equipo quirúrgico, aumentar la complejidad de sus casos. En México, la evolución de la cirugía de mínimo acceso se hace presente, permaneciendo a la vanguardia en el manejo quirúrgico.

Palabras clave: Cirugía robótica, funduplicatura, cirugía robótica en cáncer de colon, gastrectomía, colectomía, plastia inguinal, cirugía general.

\section{ABSTRACT}

Objectives: Robotic surgery is becoming more available every day and, in some cases, it surpasses other surgical approaches with better results for patients. The present study describes the experience of a single surgical group in general surgery, in two private tertiary hospitals in Mexico City. Material and methods: From January 2016 to August 2020, 230 patients with different diseases underwent robotic surgery within the specialty of general surgery, the surgeries were performed by a single qualified surgeon. Surgical results were recorded in a database. Results: 230 patients underwent robotic surgery, 44\% (102) were women and $56 \%$ (128) were men with a mean age of 56.5 years with a range of 19-88 years. Hiatal surgery was the most performed in this period, with 100 surgeries (43.5\% of the total), followed by inguinal plasties with 98 (42.6\%), ventral plasties $13(5.7 \%)$, colectomies 10 (4.3\%), cholecystectomies $4(1.7 \%)$, gastrectomies $3(1.4 \%)$, resection of duodenal lesion $1(0.4 \%)$ and restitution of intestinal transit with resection of colovaginal fistula $1(0.4 \%)$. The mean surgical time was $178.4 \mathrm{~min}$. There was only one conversion to open surgery and no major complications were reported with a morbidity of $4.7 \%$. Conclusions: Robotic surgery is being performed successfully in Mexico and with results comparable to international centers of excellence and there is a timeline in the robotic surgeon's experience that allows, as he accumulates a number of instances and experience in the surgical team, to increase the complexity of his cases. In Mexico, the evolution of minimally invasive surgery is present, remaining at the forefront of surgical management.

Keywords: Robotic surgery, fundoplication, robotic surgery in colon cancer, gastrectomy, colectomy, groin plasty, general surgery.

Citar como: Kuri OJA, Solórzano AJJ, Aguirre GMP, Galeana NFI, Luján MKI. Primeros 230 casos en cirugía general asistida por robot por un solo grupo quirúrgico en México. Rev Mex Cir Endoscop. 2020; 21 (2): 79-85. https://dx.doi.org/10.35366/98911 


\section{INTRODUCCIÓN}

La idea de crear una máquina automática capaz de realizar algunas de las tareas de las manos humanas es bastante antigua. Los orígenes de este concepto pueden ser rastreados hasta la mitología griega, las tradiciones judías, edad media y renacimiento. El término «robot», sin embargo, fue acuñado sólo en 1920 gracias al autor checo Karel Čapek que escribió la obra «R.U.R. Rossumovi Univerzální Roboti (Robots universales de Rossum)». ${ }^{1}$

En 1972, el Robot Institute of America definió un robot como «un manipulador reprogramable y multifuncional, diseñado para mover materiales, piezas, herramientas o dispositivos especializados, a través de varios movimientos programados para el desempeño de una variedad de tareas». Las siguientes décadas fueron testigo de la entrada de robots en el mundo quirúrgico.

El primer procedimiento quirúrgico asistido por robot documentado fue una biopsia percutánea del cerebro, guiada por tomografía computarizada, realizada en 1985 por Kwoh y colaboradores, empleando un PUMA (máquina universal programable para ensamblaje; Westinghouse Electric, Pittsburgh, Pensilvania). ${ }^{2}$

Sin embargo, el mundo de la cirugía robótica se modernizó y comercializó realmente cuando el sistema quirúrgico Da Vinci (desarrollado por Intuitive Surgical, Sunnyvale, CA, USA) es aprobado por la FDA en el año 2000, es el único sistema en su tipo y actualmente el más distribuido. ${ }^{3}$

A finales de 2016, se instalaron más de 3,800 plataformas Da Vinci en todo el mundo, y entre 2007 y 2013, más de 1.7 millones de procedimientos robóticos se realizaron tan sólo en los Estados Unidos.

En México, la intervención robótica ha tenido un inicio y desenlace diferente a los países más desarrollados, revelando una mayor distribución y realización de procedimientos en los últimos años. Esto conforme se han revelado las ventajas de esta tecnología internacionalmente.

La primera cirugía robótica efectuada en México fue en 1996 utilizando el robot AESOP, realizada por Adrián Carbajal, quien participó en la primera telecirugía con el proyecto Da Vinci en 1998 y en el primer estudio prospectivo aleatorizado controlado de cirugía robótica Da Vinci versus intervención laparoscópica. 4,5 En 2006, en el Hospital San José en Monterrey, Nuevo León, se realizó la primera cirugía bariátrica y la primera urológica asistidas por robot en México, las cuales consistieron en la colocación de una banda gástrica y prostatectomía, respectivamente. ${ }^{6,7}$

En otros países latinoamericanos, la operación quirúrgica robótica también ha iniciado, en Venezuela inició en 2006 de forma privada; en ese año se realizó la primera cirugía robótica en el Hospital de Clínicas Caracas. Venezuela es considerada pionera en dicha intervención robótica en Latinoamérica. ${ }^{8}$ Actualmente, algunos de los países latinoamericanos que cuentan con robot Da Vinci son Colombia, Brasil, Argentina, Chile y Venezuela, entre otros. ${ }^{9}$

En México existen algunas series de grupos quirúrgicos de múltiples especialidades que varían desde agrupaciones multidisciplinarias de centros de tercer nivel hasta grupos quirúrgicos enfocados únicamente en una subespecialidad. ${ }^{10-13}$

El siguiente estudio tiene como objetivo describir la experiencia inicial de un solo grupo quirúrgico demostrando la serie más grande en México de una sola especialidad hasta ahora representada en la literatura médica indexada, así como la seguridad, viabilidad y rentabilidad de estos procedimientos en el medio privado en la República mexicana.

\section{MATERIAL Y MÉTODOS}

Serie consecutiva de casos, compilada en un estudio analítico, observacional y descriptivo realizado en dos hospitales de la Ciudad de México, de los procedimientos realizados en el Hospital Ángeles Pedregal y Centro Médico ABC, campus Santa Fe, por un mismo grupo quirúrgico y liderado por un solo cirujano, durante el periodo comprendido entre enero de 2016 y agosto de 2020. Los procedimientos incluidos en este estudio fueron diversos, todos relacionados con una sola especialidad, la cirugía general.

La información registrada fue: tipo de procedimiento, edad, sexo, sangrado, tiempo quirúrgico y tiempo de docking. De igual manera, se llevó a cabo una catalogación y descripción de las complicaciones asociadas a dichos procedimientos. La información fue recopilada y analizada en una base de datos procesada en Microsoft Excel.

\section{RESULTADOS}

Un total de 230 pacientes fueron sometidos a cirugía robótica en la plataforma quirúrgica Da Vinci $\mathrm{Si}^{\circledR}$, por un mismo cirujano en el periodo de enero de 2016 a agosto de 2020 , de los cuales $44 \%$ (102) fueron mujeres y $56 \%$ (128) fueron hombres, cuya edad promedio era de 56.5 años, con un rango de 19-88 años.

Todos los procedimientos fueron realizados por el mismo cirujano en los dos centros quirúrgicos mencionados anteriormente, apoyado por la misma agrupación quirúrgica. La cirugía de hiato fue la más realizada en este periodo acumulando 100 intervenciones (43.5\% del total), seguida por las plastias inguinales con 98 (42.6\%), plastias ventrales 13 (5.7\%), colectomías 10 (4.3\%), colecistectomías 4 (1.7\%), gastrectomías $3(1.4 \%)$, resección de lesión duodenal 1 $(0.4 \%)$ y restitución del tránsito intestinal con resección de fístula colovaginal 1 (0.4\%) (Tabla 1). 
Tabla 1: Casos de cirugía robótica realizados entre enero de 2016 y agosto de 2020.

\begin{tabular}{|c|c|c|c|c|c|}
\hline Cirugías robóticas & $\mathrm{n}$ & $\begin{array}{l}\text { Edad promedio } \\
\text { (máx., mín.) }\end{array}$ & Género (F/M) & $\begin{array}{l}\text { Tiempo qx prom } \\
\text { Min (máx., mín.) }\end{array}$ & $\begin{array}{l}\text { Sangrado } \\
\text { prom }\left(\mathrm{cm}^{3}\right)\end{array}$ \\
\hline Funduplicaturas tipo Nissen & 100 & & & & \\
\hline Primarias & 66 & $66.3(79,21)$ & $29 / 37$ & $93.2(120,60)$ & - \\
\hline Refunduplicatura & 28 & $63.2(78,32)$ & $17 / 11$ & $124.6(190,70)$ & 15 \\
\hline Piloroplastia + REDO & 6 & $52.7(63,33)$ & $4 / 2$ & $172.1(210,120)$ & 15 \\
\hline Plastias inguinales & 98 & & & & \\
\hline Unilaterales & 73 & $55.32(88,19)$ & $25 / 48$ & $46.3(120,35)$ & - \\
\hline Bilaterales & 25 & $49.3(78,22)$ & $10 / 15$ & $72.3(135,50)$ & - \\
\hline Plastias ventrales & 13 & & & & \\
\hline TAPP & 1 & $55.4(71,35)$ & $1 / 0$ & $182(220,140)$ & 50 \\
\hline TAR & 12 & $67.9(82,45)$ & $3 / 9$ & $247.7(330,200)$ & 100 \\
\hline Colectomías & 10 & & & & \\
\hline Izquierda & 7 & $67.6(71,58)$ & $5 / 2$ & $231.3(340,195)$ & 150 \\
\hline Derecha & 1 & $63(63,63)$ & $0 / 1$ & 230 & 100 \\
\hline Izquierda + metastasectomía & 2 & $65.5(67,64)$ & $1 / 1$ & $330(350,310)$ & 300 \\
\hline Colecistectomías & 4 & $44.2(79,46)$ & $3 / 1$ & $80.3(90,45)$ & 20 \\
\hline Gastrectomías & 3 & $68.3(71,65)$ & $1 / 2$ & $240(330,200)$ & 200 \\
\hline Enucleación GIST duodenal & 1 & 37 & $1 / 0$ & 290 & 310 \\
\hline $\begin{array}{l}\text { Restitución del tránsito intestinal } \\
\text { + resección de fístula }\end{array}$ & 1 & 45 & $1 / 0$ & 310 & 300 \\
\hline Total & 230 & 56.5 & $101(44 \%) / 129(56 \%)$ & $178.4(2.9 \mathrm{~h})$ & 96.9 \\
\hline
\end{tabular}

Máx. = máximo, mín. = mínimo, F/M = femenino/masculino, qx = quirúrgico, REDO = refunduplicatura, prom = promedio, TAPP = transabdominal preperitoneal repair, $\mathrm{TAR}=$ transversus abdominis release, GIST = gastrointestinal stromal tumors.

Cirugía de hiato: de los 100 procedimientos realizados en la región anatómica del hiato esofágico, 66 (29 mujeres y 37 hombres) fueron funduplicaturas tipo Nissen primarias, 28 (17 mujeres y 11 hombres) refunduplicaturas y seis piloroplastias (4 mujeres y 2 hombres) con refunduplicatura y el tiempo promedio fue de 93.2 min (rango 120-60 min), 124.6 min (rango 190-70 min) y 172.1 min (rango 210-120 $\mathrm{min}$ ) respectivamente, el sangrado promedio de $15 \mathrm{~cm}^{3}$. Dentro de las complicaciones reportadas en las refunduplicaturas primarias se reportaron un neumotórax y una reoperación a los tres meses por migración de la funduplicatura. De estas complicaciones, el neumotórax izquierdo sucedió durante la disección de una hernia gigante con estómago intratorácico, en donde el saco herniario se encontraba adherido a la pleura izquierda, el cual fue resuelto previo a la extubación del paciente con punción en segundo espacio intercostal y maniobra de Valsalva. La reoperación por migración se debió a episodios de vómitos múltiples por intoxicación alimentaria en un paciente femenino de 41 años, a los tres meses de la operación inicial, la cual también había sido realizada mediante cirugía robótica por este mismo grupo quirúrgico, la operación se llevó a cabo sin ninguna complicación y la paciente se encuentra evidenciando excelente evolución y asintomática hasta el momento. Dentro de las refunduplicaturas como complicación se reportó un neumotórax izquierdo, durante la disección de la recidiva de la hernia en un paciente de 50 años, el cual se resolvió previo a la extubación del paciente con punción en segundo espacio intercostal y maniobra de Valsalva, sin complicaciones ni necesidad de sonda endopleural. En los procedimientos que utilizan piloroplastias con refunduplicaturas no se reportaron complicaciones.

Plastias inguinales: se realizaron 98 plastias inguinales asistidas por robot, 73 unilaterales (25 mujeres y 48 hombres) y 25 bilaterales (10 mujeres y 15 hombres), el tiempo promedio fue de $46.3 \mathrm{~min}$ (rango 120-35 min) y $72.3 \mathrm{~min}$ (rango 135-50 $\mathrm{min}$ ) respectivamente. Las edades promedio fueron de 55.32 años (rango 88-19 años) para las hernias unilaterales y 49.3 años (78-22 años) para las hernias bilaterales. Las plastias unilaterales fueron realizadas mediante técnica TAPP (transabdominal preperitoneal repair), colocando una malla de monofilamento de polipropileno y ácido poliláctico autoadherible (ProGrip ${ }^{\mathrm{TM}}$ ), derecha o izquierda dependiendo del lado a tratar. Hasta el momento, en el seguimiento que se ha dado, ninguna hernia ha recurrido, no se reportó ninguna complicación transoperatoria. Las plastias inguinales bilaterales se realiza- 
ron mediante la técnica de r-Stoppa modificado, con una sola malla que cubriera ambos defectos $y$, dependiendo del caso (recurrente o no recurrente), se realizó un colgajo peritoneal único con descenso vesical o dos colgajos separados con un comunicante posterior a la vejiga para el paso de la malla. La malla utilizada es de material mixto de polipropileno y poliglecaprone $\left(\right.$ UltraPro $^{\mathrm{TM}}$ ) de $30 \times$ $30 \mathrm{~cm}$, la cual era cortada a la medida exacta del colgajo peritoneal en transversal y que cubriera los orificios miopectíneo, indirecto, directo y femoral.

Plastias ventrales: se realizaron 13 plastias ventrales asistidas por robot, 1 TAPP (transabdominal preperitoneal repair) y 12 TAR (transversus abdominis release). Éstas fueron ejecutadas en una mujer para la plastia tipo TAPP y tres mujeres y cinco hombres para la plastia tipo TAR con tiempos quirúrgicos promedios de $182 \mathrm{~min}$ (rango 220$140 \mathrm{~min}$ ) y $247.7 \mathrm{~min}$ (rango $330-200 \mathrm{~min}$ ) cuyas edades promedio eran de 67.9 años (rango 82-45 años) y 55.4 (rango 71-35 años) respectivamente. La malla utilizada es una malla mixta de polipropileno y poliglecaprone (UItraPro $^{\mathrm{TM}}$ ) de $30 \times 30 \mathrm{~cm}$, la cual era cortada a la medida exacta para cubrir el área completa de disección. La única complicación hasta ahora descrita en estos pacientes fue la presencia en dos eventos de hematoma posoperatorio que requirió drenaje por radiología intervencionista y colocación de drenaje cerrado, el cual fue retirado posterior a una semana, en ambas oportunidades sin mayores complicaciones. La cobertura antimicrobiana se continuó en ambos casos hasta tres días posteriores al retiro del drenaje.

Colectomías: dentro de las colectomías asistidas por robot se realizaron en este periodo 10 procedimientos, los cuales fueron una colectomía derecha ( 1 hombre), siete colectomías izquierdas ( 5 mujeres y 2 hombres) y dos hemicolectomías izquierdas con metastasectomías hepáticas (1 mujer y 1 hombre) con tiempos quirúrgicos promedios de $230 \mathrm{~min}, 231.3 \mathrm{~min}$ (rango 340-195 min) y $330 \mathrm{~min}$ (rango 350-310 min) respectivamente y edades promedio de 63 años para la colectomía derecha, 67.6 años (rango 71-58 años) para las colectomías izquierdas y 65.5 años (67-64 años) para el tercer tipo. Éste es, dentro de las intervenciones realizadas por este grupo quirúrgico, probablemente el conjunto de operaciones más heterogéneo de todas las incluidas en esta serie, puesto que se incluyen cirugías de patología inflamatoria, oncológica y diferentes áreas anatómicas, sin embargo, al tratarse de un mismo tipo de intervención y para la exposición de esta serie fueron agrupadas en la misma categoría quirúrgica. La hemicolectomía derecha fue una resección oncológica adecuada, corroborada por patología, al tratarse de un adenocarcinoma de colon derecho con una reconstrucción ileotransverso anastomosis latero-lateral intracorpórea usando una engrapadora lineal $\left(\mathrm{ECHELON}^{\mathrm{TM}}\right)$ y el cierre de la brecha usando sutura barbada. Las hemicolectomías izquierdas se dividieron en sigmoidectomías por enfermedad diverticular, ${ }^{4}$ a las cuales se les realizó una anastomosis con engrapadora circular colorrectal término-terminal, y hemicolectomías izquierdas oncológicas en donde, dependiendo el nivel de la resección, se valoró una anastomosis colorrectal con engrapadora circular utilizando la técnica de Vijan-POP totalmente intracorpórea o una anastomosis colo-colo latero-lateral como se describió previamente. En cuanto a los procedimientos combinados con metastasectomía hepática se realizaron dos dockings, uno para el procedimiento de colon con la técnica descrita previamente y el segundo para la metastasectomía hepática, resultando un total de dos dockings y seis puertos para el procedimiento completo. De los 10 procedimientos se realizó solamente una conversión a operación abierta en uno de los pacientes con antecedente de enfermedad diverticular por falta de progresión en la disección y dificultad en la técnica quirúrgica; se decidió la conversión por seguridad del paciente. Se completó el procedimiento de manera abierta sin ninguna complicación.

Colecistectomías: cuatro colecistectomías se realizaron mediante asistencia robótica. De estos cuatro casos, tres fueron mujeres y uno hombre con tiempo quirúrgico promedio de $44.2 \mathrm{~min}$ (rango $79-46 \mathrm{~min}$ ), y edad promedio de 44.2 años (rango 79-46 años), no se describieron complicaciones.

Gastrectomías: se realizaron tres gastrectomías asistidas por robot. Los procedimientos realizados fueron: la resección de un tumor de GIST de la unión esofagogástrica (1 hombre) y dos esofagectomías distales con gastrectomía proximal con esofagogastro anastomosis y disección ganglionar D1 y piloroplastia por adenocarcinoma de la unión esofagogástrica, dando respuesta adecuada a la neoadyuvancia ( 1 hombre y 1 mujer). El tiempo quirúrgico promedio fue de $240 \mathrm{~min}$ (rango 330-200 min). Ninguna complicación mayor fue registrada, uno de los pacientes con esofagectomía distal y gastrectomía proximal desarrolló disfagia leve, la cual fue tratada mediante dieta y manejo médico con resolución.

Enucleación de GIST duodenal: se realizó una enucleación de un GIST duodenal en una paciente femenina de 37 años de edad con un tiempo quirúrgico de 290 minutos sin ninguna complicación, con alta tolerando dieta al día cuatro posoperatorio.

Restitución del tránsito intestinal con resección de fístula colovaginal: un procedimiento de restitución de tránsito intestinal de un paciente femenino de 45 años portadora de colostomía y con una fístula colovaginal y adecuado estado nutricional, con antecedente de histerectomía laparoscópica, como complicación a procedimiento ginecológico. Se realizó la restitución del tránsito intestinal 
con colorrecto anastomosis término-terminal utilizando engrapadora circular y la resección de una fístula con cierre primario. El tiempo quirúrgico fue de 310 min y no se reportaron complicaciones. La paciente fue dada de alta al día posoperatorio siete, tolerando la vía oral y con evacuaciones.

Tiempo de docking: el tiempo de docking promedio en las cirugías es de 7 minutos, sin embargo, varía entre el tipo de operaciones, llegando a ser de 4 minutos en las plastias inguinales, de 6 minutos en las funduplicaturas y de 8 minutos en las colectomías.

\section{DISCUSIÓN}

La primera serie que se encontró en la revisión bibliográfica de la literatura mexicana es el conjunto de casos atendido en el Hospital Regional de Alta Especialidad de Zumpango por el programa de Cirugía Robótica entre 2013 y 2015, en donde relatan su experiencia inicial, describiendo 99 pacientes a los que se les realizaron un total de 102 procedimientos de diferentes especialidades, ejecutadas por ocho cirujanos diferentes, incluyendo urología (48), cirugía gastrointestinal (43) y ginecología (11 casos), siendo ésta la serie de mayor número en la literatura de este país hasta el momento. ${ }^{10}$

En cuanto a las series de especialidades únicas, la casuística disminuye de manera importante por especialidad e incluso por tipo de padecimiento. Durante el 2015, el Dr. Villanueva y colegas publicaron una serie de cinco casos como experiencia inicial en cirugía colorrectal en México. Por otro lado, el Dr. Cuendis y colaboradores han plasmado diversas publicaciones enfocadas hacia la cirugía robótica en la subespecialidad hepatopancreatobiliar, en las que destacan dos series comparando la operación laparoscópica contra la robótica en fístula bilioentérica posterior a lesión de vía biliar durante colecistectomía (5 casos) y reparación de la vía biliar y hepático-yeyuno anastomosis (35 casos) donde el número de eventos disminuye dada la baja incidencia de la patología. ${ }^{11-13}$

Relacionados con la cirugía del hiato esofágico, existe un reporte por parte del Dr. Santamaría y asociados, de 18 funduplicaturas tipo floppy Nissen en el periodo de 20142017, en donde se realizó un estudio de seguimiento para valorar la escala de Visick en donde se lograron, en el $84 \%$ de sus pacientes, la remisión de los síntomas y ninguno de ellos en un Visick IV (aumento de los síntomas). ${ }^{14}$

La primera intervención realizada por este grupo quirúrgico fue una colecistectomía en 2016 y posteriormente se continuaron con procedimientos menores como hernias inguinales y funduplicaturas tipo Nissen hasta mediados de 2017 en donde, posterior al aumento de los casos para completar la adaptación del grupo quirúrgico a la cirugía robótica, se realizó la primera operación mayor que fue una sigmoidectomía por enfermedad diverticular, la cual se realizó sin complicaciones. Al cabo de cuatro años en esta serie, se reportaron 230 casos atendidos por un mismo grupo quirúrgico y una sola especialidad.

Como se comentó previamente, la primera intervención realizada en 2016 fue una colecistectomía, de las cuales se realizaron cuatro en total, siendo la última en 2019. Sin embargo, como comentaron Bhakavi y colaboradores en su revisión de 91,849 pacientes sometidos a colecistectomía asistida por robot en Estados Unidos, este procedimiento es seguro pero costoso $y$, en la experiencia del equipo mexicano autor de este estudio, no tiene en este momento ninguna ventaja real sobre la laparoscopía convencional. ${ }^{15}$

En la cirugía de colon, el abordaje robótico ha revolucionado el panorama actual, como reportan diversas series en donde se ha disminuido significativamente la morbilidad, menor estancia hospitalaria e incidencia de conversión, de $7.5 \%$ en cirugía robótica a 14\% en cirugía laparoscópica, cuando es comparada en enfermedad diverticular. ${ }^{16}$ En cirugía oncológica de colon, de igual manera se reportan resultados oncológicos equiparables a la operación abierta o laparoscópica, con menores porcentajes de conversión, mayor promedio de ganglios resecados, así como menor estancia hospitalaria. ${ }^{17}$

Estas estadísticas son comparables con las encontradas en los pacientes de la serie presentada, al encontrar únicamente una conversión $(10 \%)$ y ninguna otra complicación u otra morbilidad asociada, al contrario, exhibiendo una recuperación acelerada de los pacientes y un mejor pronóstico general contrastado con las otras técnicas quirúrgicas. En este momento, los números mostrados aquí en cuanto a las colectomías asistidas por robot representan una serie escasa y se espera completar a futuro un mayor número con seguimiento prospectivo para generar una estadística significativa.

La técnica quirúrgica robótica para plastias inguinales ha sido adoptada con mayor frecuencia a nivel internacional en los últimos años y en la literatura se reportan ambas técnicas quirúrgicas (r-TAPP y r-Stoppa modificada), una tasa baja de complicaciones intraoperatorias y baja tasa de conversión $(0.03 \%$ y $1.4 \%$ respectivamente), en el posoperatorio también se asocia con tasas bajas de retención urinaria y seroma/hematoma (4.1\% y 3.5\% respectivamente), finalmente, la tasa de recurrencia es de $0.18 \%$. En esta serie de casos, sólo siete pacientes presentaron seroma en el puerto umbilical $(7.14 \%)$, no se presentaron otro tipo de complicaciones transoperatorias $y$, en el seguimiento posoperatorio a siete días, 30 días, seis meses y un año se tiene una tasa de recurrencia del $0 \%$ hasta el momento. ${ }^{18,19}$

En cuanto al abordaje asistido por robot para realizar una funduplicatura empieza a reflejar resultados superiores a los obtenidos por laparoscopía; Marzio Frazzoni y colegas en 
2012, en un estudio de 44 pacientes en el grupo laparoscópico y 44 en el grupo robótico, documentan una mayor disminución de valores anormales del tiempo de exposición esofágica al ácido al ser comparado frente al abordaje laparoscópico convencional, $14 \%$ de los casos en el grupo laparoscópico contrastado con cero eventos en el grupo robótico. ${ }^{20}$

El procedimiento de tipo REDO (refunduplicatura) se describe en la literatura médica como un procedimiento con alta morbilidad perioperatoria $(7.3-9.9 \%),{ }^{21,22}$ el abordaje asistido por robot permite al cirujano una mayor precisión en el manejo de los tejidos ya modificados por los procedimientos previos, disminuyendo la morbilidad perioperatoria asociada a la cirugía. Alexander C. Mertens y Rob C. Tolboom, en una revisión retrospectiva de 151 procedimientos de REDO, documentan la incidencia de $2.6 \%$ para complicaciones mayores en el periodo perioperatorio. ${ }^{23}$ En la serie aquí presentada, se documentó sólo una complicación no grave (3.5\%) en el grupo de REDO que se resolvió sin reintervención quirúrgica.

El tiempo quirúrgico de una funduplicatura primaria asistida por robot es documentado entre 88 a 137 minutos. ${ }^{24}$ En la serie en estudio, el tiempo para un procedimiento primario fue de 60 a 120 minutos (promedio de 93.2 minutos), encontrándose dentro los tiempos que manejan los centros de referencia y alta especialidad internacionales. Es importante mencionar que el acoplamiento lateral (side docking) permite un libre acceso a la vía aérea y al control endoscópico que se realiza en el transoperatorio.

La gastrectomía robótica ha sido implementada de mayor manera en los años recientes, e incluso en países como Japón, con uno de los índices más altos de cáncer gástrico, fue autorizado que esta enfermedad tuviera cobertura de seguridad nacional en abril de 2018. En múltiples series se demuestra la seguridad de esta técnica y su superioridad sobre la abierta y la laparoscópica para la resección oncológica del cáncer gástrico, disminuyendo morbilidad, sangrado transoperatorio y estancia posoperatoria, sin embargo, sí aumentan los costos quirúrgicos comparados frente a las cirugías abierta y laparoscópica. ${ }^{25,26}$

Los tres casos de gastrectomías reportados en esta serie son escasos para ser representativos estadísticamente; sin embargo, los resultados son comparables con los aquellos reportados en la literatura internacional y hasta el momento no se ha presentado ninguna complicación mayor.

La resección del GIST duodenal data de 2019 y fue una enucleación llevada a cabo sin complicaciones. Las series de esta patología a nivel nacional e internacional son escasas, al ser una de mucho menor incidencia, sin embargo, en las series de operaciones descritas se está evidenciando seguridad para el paciente y adecuados resultados oncológicos, sin embargo, se requieren mayores estudios para valorar el provecho real a largo plazo y el costo-beneficio. ${ }^{27,28}$
En cuanto a la restitución del tránsito intestinal y resección de fístula colovaginal realizada a una paciente de 45 años, es un caso único. Esto demuestra que la cirugía asistida por robot puede ser utilizada incluso en abdómenes hostiles o con intervenciones previas. Existen múltiples reportes de reparaciones asistidas por robot de fístulas rectovaginales por enfermedad diverticular o incluso fístulas colovesicales. ${ }^{29,30}$

Sin embargo, en este caso fue realizado el desmantelamiento de una colostomía y restitución de tránsito intestinal con resección de fístula con cierre primario sin ninguna complicación.

\section{CONCLUSIÓN}

La cirugía robótica se está realizando exitosamente en México y ofrece resultados comparables a los centros de excelencia internacional y existe una línea del tiempo en la experiencia del cirujano robótico que permite, conforme acumula un número de eventos y experiencia en el equipo quirúrgico, aumentar la complejidad de sus casos.

En México, la evolución de la cirugía de mínimo acceso se hace presente, permaneciendo a la vanguardia en el manejo quirúrgico. Es satisfactorio poder contribuir a la literatura médica y de esta forma impulsar el desarrollo del conocimiento de esta tecnología en las nuevas generaciones en las distintas especialidades quirúrgicas. Se concluye que el abordaje robótico es una herramienta que mejora el pronóstico y disminuye la morbilidad asociada al procedimiento. Tras el objetivo de generar información estadísticamente significativa, se debe generar mayor evidencia con estudios prospectivos en México y el mundo que sustenten los beneficios de la cirugía robótica.

\section{REFERENCIAS}

1. Čapek K. The meaning of R.U.R. Saturday Rev. 1923; 136: 79.

2. Kwoh YS, Hou J, Jonckheere EA, Hayati S. A robot with improved absolute positioning accuracy for CT guided stereotactic brain surgery. IEEE Trans Biomed Eng. 1988; 35: 153-160.

3. Kalan S, Chauhan S, Coelho RF, Orvieto MA, Camacho IR, Palmer KJ et al. History of robotic surgery. J Robot Surg. 2010; 4: 141-147.

4. Miller H. Cirugía robótica en México. Los sistemas inteligentes, perspectivas actuales y a futuro en el ámbito mundial. Rev Mex Cir Endoscop. 2003; 4: 45-50.

5. Carbajal A. Estado del arte en cirugía robótica. Rev Mex Cir Endoscop. 2001; 2: 109-112.

6. González RR, Ballí JJ, Rumbaut RD. Nuevas tecnologías en cirugía: cirugía robótica. Avances. 2007; 13: 41-50.

7. Corona-Montes VE. La cirugía robótica como alternativa para el tratamiento del cáncer de próstata de bajo riesgo en México. Rev Mex Urol. 2013; 73: 221-222. 
8. Iranmanesh P, Morel P, Wagner OJ, Inan I, Pugin F, Hangen ME. Set-up and docking of the da Vinci surgical system: prospective analysis of initial experience. Int J Med Robot. 2010; 6: 57-60.

9. Lucena OJR, Coronel P, Orellana PS. Historia, evolución, estado actual y futuro de la cirugía robótica. RFM. 2007; 30: 109-114.

10. Ruiz Suárez E, Ortiz Wong R, Rodríguez Reyes G, Valadez Caballero D, Blanco Figueroa J, González Santamaría J. Experiencia inicial en cirugía robótica mínimamente invasiva en hospital de tercer nivel en México. Rev Mex Cir Endoscop. 2016; 17: 29-33.

11. Villanueva-Sáenz E, Ramírez-Ramírez MM, ZubietaO’Farrill G, García-Hernández L. Experiencia inicial en cirugía colorrectal asistida por robot en México. Cir Cir. 2017; 85: 284-291.

12. Cuendis-Velázquez A, Trejo-Ávila M, Rodríguez-Parra A, Bada-Yllán O, Morales-Chávez C, Fernández-Álvarez L et al. Minimally invasive approach (robotic and laparoscopic) to biliary-enteric fistula secondary to cholecystectomy bile duct injury. J Robot Surg. 2018; 12: 509-515.

13. Cuendis-Velázquez A, Trejo-Ávila M, Bada-Yllán O, Cárdenas-Lailson E, Morales-Chávez C, Fernández-Álvarez $L$ et al. A new era of bile duct repair: robotic-assisted versus laparoscopic hepaticojejunostomy. J Gastrointest Surg. 2019; 23: 451-459.

14. González-Santamaría JR, Valderrama Gutiérrez MR, Rubio Arroyo EH, Flores Rangel GA. Current status of the robotic fundoplication: case series and literature review. Ann Clin Case Rep. 2018; 3: 1548.

15. Pokala B, Flores L, Armijo PR, Kothari V, Oleynikov D. Robot-assisted cholecystectomy is a safe but costly approach: A national database review. Am J Surg. 2019; 218: 1213-1218. doi: 10.1016/j.amjsurg.2019.08.014.

16. Al-Temimi MH, Chandrasekaran B, Agapian J, Peters WR Jr, Wells KO. Robotic versus laparoscopic elective colectomy for left side diverticulitis: a propensity scorematched analysis of the NSQIP database. Int J Colorectal Dis. 2019; 34: 1385-1392. doi: 10.1007/s00384-01903334-x.

17. Dolejs SC, Waters JA, Ceppa EP, Zarzaur BL. Laparoscopic versus robotic colectomy: a national surgical quality improvement project analysis. Surg Endosc. 2017; 31 : 2387-2396. doi: 10.1007/s00464-016-5239-5.

18. Charles EJ, Mehaffey JH, Tache-Leon CA, Hallowell PT, Sawyer RG, Yang Z. Inguinal hernia repair: is there a benefit to using the robot? Surg Endosc. 2018; 32: 2131-2136.
19. Arcerito M, Changchien E, Bernal O, Konkoly-Thege A, Moon J. Robotic inguinal hernia repair: technique and early experience. Am Surg. 2016; 82: 1014-1017.

20. Frazzoni M, Conigliaro R, Colli G, Melotti G. Conventional versus robot-assisted laparoscopic Nissen fundoplication: a comparison of postoperative acid reflux parameters. Surg Endosc. 2012; 26: 1675-1681. doi: 10.1007/s00464-011-2091-5.

21. Nageswaran H, Haque A, Zia M, Hassn A. Laparoscopic redo anti-reflux surgery: case-series of different presentations, varied management and their outcomes. Int J Surg. 2017; 46: 47-52. doi: 10.1016/j.ijsu.2017.08.553.

22. Singhal S, Kirkpatrick DR, Masuda T, Gerhardt J, Mittal SK. Primary and redo antireflux surgery: outcomes and lessons learned. J Gastrointest Surg. 2018; 22: 177-186. doi: 10.1007/s11605-017-3480-4.

23. Mertens AC, Tolboom RC, Zavrtanik H, Draaisma WA, Broeders IAMJ. Morbidity and mortality in complex robotassisted hiatal hernia surgery: 7-year experience in a highvolume center. Surg Endosc. 2019; 33: 2152-2161. doi: 10.1007/s $/ \mathrm{s}^{0} 0464-018-6494-4$.

24. Zhang P, Tian JH, Yang KH, Li J, Jia WQ, Sun SL et al. Robotassisted laparoscope fundoplication for gastroesophageal reflux disease: a systematic review of randomized controlled trials. Digestion. 2010; 81: 1-9. doi: 10.1159/000235920.

25. Alhossaini RM, Altamran AA, Seo WJ, Hyung WJ. Robotic gastrectomy for gastric cancer: Current evidence. Ann Gastroenterol Surg. 2017; 1: 82-89. doi: 10.1002/ ags3.12020.

26. Chen K, Pan Y, Zhang B, Maher H, Wang XF, Cai XJ. Robotic versus laparoscopic Gastrectomy for gastric cancer: a systematic review and updated meta-analysis. BMC Surg. 2017; 17: 93. doi: 10.1186/s²893-017-0290-2.

27. Vicente E, Quijano Y, Ielpo B, Duran H, Diaz E, Fabra I et al. Robot-assisted resection of gastrointestinal stromal tumors (GIST): a single center case series and literature review. Int J Med Robot. 2016; 12: 718-723.

28. Downs-Canner S, Van der Vliet WJ, Thoolen SJ, Boone BA, Zureikat AH, Hogg ME et al. Robotic surgery for benign duodenal tumors. J Gastrointest Surg. 2015; 19: 306-312. doi: 10.1007/s11605-014-2668-0.

29. Puntambekar S, Rayate N, Agarwal G, Joshi S, Rajmanickam S. Robotic rectovaginal fistula repair. J Robot Surg. 2012; 6: 251-253. doi: 10.1007/s11701-011-0282-7.

30. Xia J, Paul Olson TJ, Rosen SA. Robotic-assisted surgery for complicated and non-complicated diverticulitis: a singlesurgeon case series. J Robot Surg. 2019; 13: 765-772. doi: 10.1007/s11701-018-00914-x. 